\title{
Spatial Mode Matching Efficiencies for Heterodyned GaAlAs Semiconductor Lasers
}

\author{
K. A. WINICK AND P. KUMAR
}

\begin{abstract}
High spatial mode matching efficiencies are experimentally demonstrated when open-loop frequency-stabilized GaAlAs semiconductor lasers are heterodyned on silicon p-i-n detectors. Repeatable values of 75 percent are obtained using two independent lasers. The mode matching efficiency increases to 90 percent when a single modulated laser is self-heterodyned using an unequal path length MachZehnder interferometer. Experimental data is shown to match closely with theoretical predictions.
\end{abstract}

\section{INTRODUCTION}

$\mathrm{T}$ HE PERFORMANCE of an optical heterodyne receiver will be strongly influenced by the degree to which the signal and local oscillator (LO) beams can be spatially matched in both amplitude and phase at the detector. Any mismatch will reduce the IF signal power and consequently the signal-to-noise ratio. The ratio of IF signal power obtained in the presence of a mismatch to that obtained when there is no mismatch will hereafter be referred to as the spatial mode matching efficiency (MME).

With the advent of efficient single-mode semiconductor lasers, free-space optical heterodyne communications between satellites is reaching the stage of practicality. In fact, such a system is currently being developed by M.I.T. Lincoln Laboratory and launch is expected before the end of the decade [1]. The successful completion of this project requires that high spatial mode matching efficiencies be realized.

Several authors [2]-[7] have performed theoretical studies to determine the MME as a function of system parameters, including signal/LO beam angular misalignment, signal/LO beam size differences, and detector-tospot size ratios. Experiments to quantitatively measure mode matching efficiencies, however, are few. Sonntag [8] has measured the functional dependence of the MME versus signal/LO angular misalignment. In his experiments, the signal beam was a plane wave and the LO was either a plane or spherical wave. The experimental results obtained had the functional form predicted by theory, but absolute MME values were not reported. In addition, the experiments were performed with a $\mathrm{HeNe}$ laser, rather than a semiconductor device, and only self-heterodyning performance on a photomultiplier tube was measured.

We have experimentally measured the MME when two independent GaAlAs semiconductor lasers are heterodyned on a high speed silicon $p-i-n$ photodetector. The experiments have also been repeated using a single, frequency-modulated GaAlAs laser, which is self-heterodyned with the aid of an unequal path length Mach-Zehnder interferometer. In the former case, maximum repeatable MME values of 75 percent were obtained. In the later case, these values increased to approximately 90 percent.

Section II of this paper gives mathematical expressions for spatial mode matching efficiencies. Gaussian signal and LO beams are assumed, and losses due to both angular misalignment and lateral offsets between beams are included. An upper bound for the loss produced by small wavefront differences between beams is also derived. The main core of this paper is contained in Section III, where our experimental setup is described and our data is analyzed. It is shown that theoretical predictions, based on the expressions of Section II, agree well with the data. Finally, Section IV summarizes our results.

\section{Mathematical Expressions for MME}

The mode matching efficiency of a heterodyne system is given by [3]:

$$
\mathrm{MME}=\frac{\left|\iint A_{S}(x) \exp \left[i \phi_{S}(x)\right] A_{\mathrm{LO}}(x) \exp \left[-i \phi_{\mathrm{LO}}(x)\right] d x\right|^{2}}{\iint A_{S}^{2}(x) d x \iint A_{\mathrm{LO}}^{2}(x) d x}
$$

Manuscript received April 27, 1987; revised August 24, 1987.

K. A. Winick is with Lincoln Laboratory, Massachusetts Institute of Technology, Lexington, MA 02173

P. Kumar was with Lincoln Laboratory, Massachusetts Institute of Technology, Lexington, MA 02173. He is now with Northwestern University, Evanston, IL 60201 .

IEEE Log Number 8718501. where $A_{S}(\boldsymbol{x})$ and $A_{\mathrm{LO}}(\boldsymbol{x})$ are the amplitude of the signal and local oscillator beams, respectively. Similarly, $\phi_{S}(x)$ and $\phi_{\mathrm{LO}}(\boldsymbol{x})$ are the beam phases. The integrals in (1) are performed over the detector area. Equivalently, they may be performed over any plane lying along the common signal/LO propagation paths. For simplicity, the plane will 
be chosen to lie at the front surface of a lens located one focal length in front of the detector [9]. It will also be assumed that the detector area is much larger than the focused spots, and that the detector has uniform quantum efficiency across its surface.

We first consider the case where the signal and LO beams at the lens are Gaussian in amplitude and flat in phase with a relative angular tilt of $\Delta \theta$ rad between their propagation directions and a lateral displacement of $\epsilon$ meters between their centers. The nominal wavelength is $\lambda$. The distance of the $1 / e$ power points measured from the beam center will be designated by $\sigma_{\mathrm{LO}}$ and $\sigma_{S}$ for the LO and signal beam, respectively. Then using (1), it is straightforward to show that

$$
\begin{aligned}
\mathrm{MME}= & \frac{4\left(\frac{\sigma_{\mathrm{LO}}}{\sigma_{S}}\right)^{2}}{\left[1+\left(\frac{\sigma_{\mathrm{LO}}}{\sigma_{S}}\right)^{2}\right]^{2}} \exp \left[\frac{-\left(\frac{\epsilon}{\sigma_{S}}\right)^{2}}{1+\left(\frac{\sigma_{\mathrm{LO}}}{\sigma_{S}}\right)^{2}}\right] \\
& \cdot \exp \left[\frac{-4 \pi^{2} \sigma_{\mathrm{LO}}^{2}\left(\frac{\sin \Delta \theta}{\lambda}\right)^{2}}{1+\left(\frac{\sigma_{\mathrm{LO}}}{\sigma_{S}}\right)^{2}}\right] .
\end{aligned}
$$

It can be seen from (2) that the MME due to tilt alone (i.e., $\epsilon=0$ and $\sigma_{\mathrm{LO}}=\sigma_{S}$ ) is given by

$$
\mathrm{MME}_{\mathrm{TILT}}=\exp \left[-2 \pi^{2} \sigma_{\mathrm{LO}}^{2}\left(\frac{\sin \Delta \theta}{\lambda}\right)^{2}\right]
$$

that the MME due to lateral displacement alone (i.e., $\Delta \theta$ $=0$ and $\sigma_{\mathrm{LO}}=\sigma_{S}$ ) is given by

$$
\mathrm{MME}_{\mathrm{LAT}}=\exp \left[-0.5\left(\frac{\epsilon}{\sigma_{S}}\right)^{2}\right]
$$

and finally that the MME due to beam size differences alone (i.e., $\epsilon=0$ and $\Delta \theta=0$ ) is given by

$$
\mathrm{MME}_{\mathrm{SIZE}}=\frac{4\left(\frac{\sigma_{\mathrm{LO}}}{\sigma_{S}}\right)^{2}}{\left[1+\left(\frac{\sigma_{\mathrm{LO}}}{\sigma_{S}}\right)^{2}\right]^{2}}
$$

Figs. 1, 2, and 3 plot the mode matching loss (i.e., 10 $\log$ MME) for tilt, lateral offset, and beam size differences, respectively, as a function of $\sigma_{\mathrm{LO}} \sin \Delta \theta / \lambda, \epsilon$ and $\sigma_{\mathrm{LO}} / \sigma_{S}$. Note the relative insensitivity of the MME to lateral offsets and beam size variations. Conversely, the MME is extremely sensitive to tilt. In the experiment to be described in Section III, $\sigma_{\text {LO }} \approx 1.84 \mathrm{~mm}$ and $\lambda \approx 0.83$ $\mu \mathrm{m}$. Thus, $\Delta \theta=50 \mu \mathrm{rad}$ will result in a $1-\mathrm{dB}$ loss.

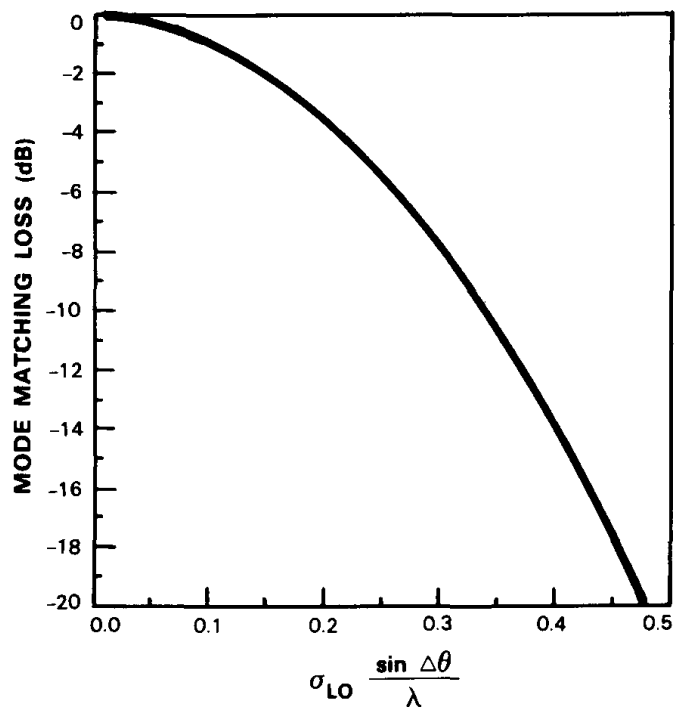

Fig. 1. Mode matching loss as a function of tilt.

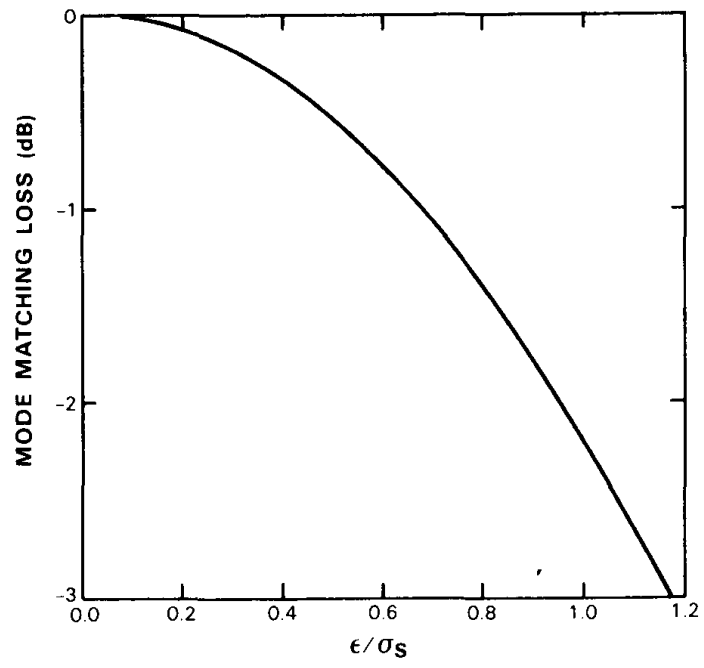

Fig. 2. Mode matching loss as a function of lateral offset.

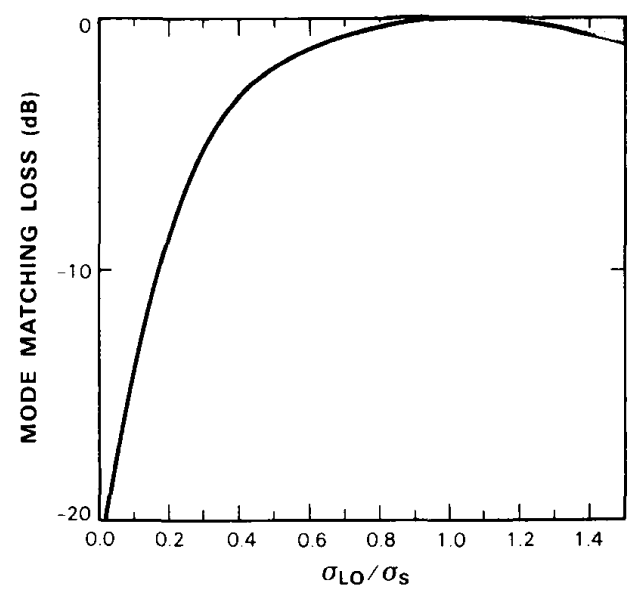

Fig. 3. Mode matching loss as a function of beam size ratio. 
Next we consider the case where the signal and LO beams at the lens, which lies one focal length in front of the detector, are matched in amplitude (i.e., $A_{S}(\boldsymbol{x})=$ $\left.A_{\mathrm{LO}}(\boldsymbol{x})\right)$ but not in phase. It is also assumed that the phase mismatch is small or equivalently:

$$
\left|\phi_{S}(x)-\phi_{\mathrm{LO}}(x)\right| \ll 1 \text {. }
$$

In addition, since (1) is invariant to an additive phase constant (i.e., path length delay) in either the signal or LO beam, we will require without loss of generality that:

$$
\iint\left(\phi_{S}(x)-\phi_{\mathrm{LO}}(\boldsymbol{x})\right) d x=0 .
$$

Then if $A_{S}(x)=$ a constant, it is easy to show by (1), (6), and (7) that

$$
\mathrm{MME}_{\mathrm{PHASE}}=1-\frac{\iint\left[\phi_{S}(x)-\phi_{\mathrm{LO}}(x)\right]^{2} d x}{\iint d x} .
$$

Noting that the mean-squared phase error (MSE) between the two beams is simply the second term on the right-hand side of (8) above, it follows that

$$
\mathrm{MME}_{\mathrm{PHASE}} \approx 1-\mathrm{MSE}, \quad \text { provided MSE } \ll 1 .
$$

Furthermore, it is shown in Appendix A that for arbitrary $A_{S}(\boldsymbol{x})$, (9) becomes

$$
\mathrm{MME}_{\mathrm{PHASE}} \geq 1-\mathrm{MSE}, \text { provided MSE } \ll 1 \text {. }
$$

\section{Experimental Setup}

Fig. 4 shows the experimental setup used to measure mode matching efficiency. In Fig. 4 the output of a Hitachi CSP 15-mW GaAlAs semiconductor laser is passed through an $\mathrm{F} / 1.177-\mathrm{mm}$ focal length collimating lens followed by an anamorphic prism pair. The prism pair serves to circularize the elliptical shape of the output beam. The circularized output profile has an approximately Gaussian intensity distribution with the $1 / e$ intensity point lying $1.84 \mathrm{~mm}$ from the beam center. The collimated laser beam is next split at beamsplitter 1 and then sent through an unequal path length Mach-Zehnder interferometer where the two beams recombine at beamsplitter 4. Fifty percent of the combined beam is picked off at beamsplitter 5 where it is sent to a commercial beam scan device and a commercial laser diode wavefront measuring instrument (LADITE) [10]. The beam scan device produces intensity profiles of either beam 1 or beam 2 by scanning a $10-\mu \mathrm{m}$-wide slit across the beam while displaying the collected power on an oscilloscope. The LADITE is a computer-controlled Mach-Zehnder interferometer which can analyze laser beam wavefronts. Its capabilities include, but are not limited to, the ability to subtract the phase of two wavefronts point by point, fit Zernike polynomials to the resulting difference, and compute the mean-squared phase value of the difference. Thus

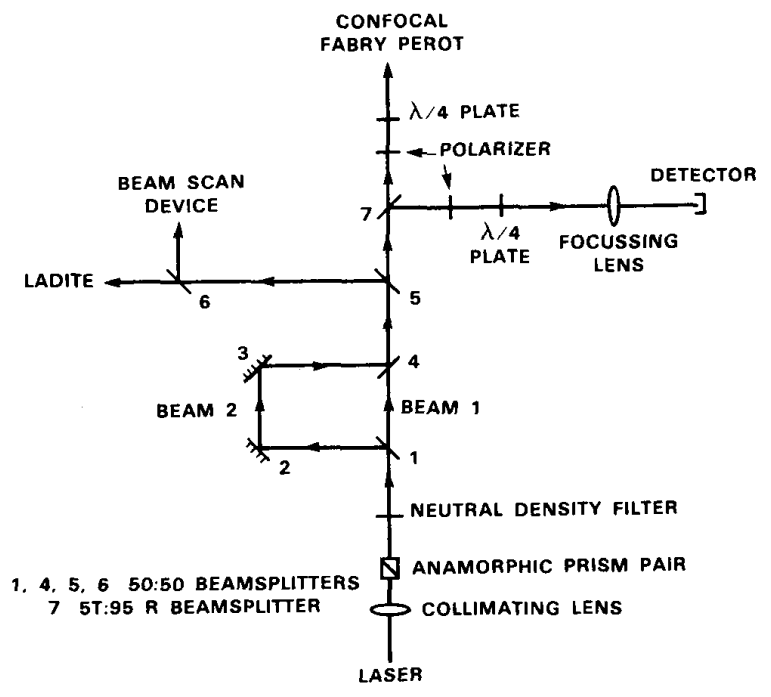

Fig. 4. Single laser experimental setup.

the LADITE in Fig. 4 can be used to measure angular tilt $(\Delta \theta)$ between the two beams at beamsplitter 4 .

In principle, the LADITE could be used to analytically calculate the mode matching efficiency as given by (1). There are, however, two practical limitations which preclude this option. First, because of unwanted multiple reflections inside the LADITE, the instrument is not capable of accurately measuring the intensity profile of a coherent beam. Second, the signal dynamic range over which the LADITE can measure phase is limited. Thus, with a Gaussian beam, the instrument fails to determine the phase in the low intensity tails of the beam. According to manufacturer specifications, the LADITE has an rms accuracy of $\lambda / 50$.

The fifty percent of the combined beams which is not sent to either the LADITE or beam scan device is further divided at beamsplitter 7 . Here, 5 percent is directed to a confocal Fabry-Perot spectrum analyzer having a 300$\mathrm{MHz}$ free spectral range and 95 percent is sent through a 25-mm focal length $\mathrm{F} / 1$ lens. A polarizer followed by a $\lambda / 4$-waveplate is placed in each of the two paths to isolate the laser from specular backscatter. An RCA high speed detector $(0.5-\mathrm{mm}$ diameter $)$ is located at the focal plane of the lens where the heterodyning takes place.

The confocal Fabry-Perot is used to coalign beams 1 and 2 . This is accomplished by blocking beam 2 and adjusting the angular and lateral position of the Fabry-Perot so that beam 1 lies directly on the axis of the Fabry-Perot. When this condition occurs the free spectral range of the Fabry-Perot doubles to $600 \mathrm{MHz}$, as observed by noting that the amplitude of every other line in the Fabry-Perot output spectrum diminishes to nearly zero [11]. Once this is accomplished beam 1 is blocked and the angular positions of mirrors 2 and 3 are adjusted so that the free spectral range of the Fabry-Perot remains at $600 \mathrm{MHz}$. In practice, we are able to coalign the two beams so that alternate lines in the Fabry-Perot output spectrum have ratios exceeding $100: 1$. 
The laser shown in Fig. 4 is open-loop frequency stabilized by controlling its temperature and its bias current. The temperature and bias currents are held constant to better than $0.0005^{\circ} \mathrm{C}$ and $1 \mu \mathrm{A}$, respectively. The laser's full-width-half maximum Lorentzian linewidth when operating at $15 \mathrm{~mW}$ is approximately $7 \mathrm{MHz}$. The laser is frequency modulated by adding a small amount of timevarying injection current to the laser bias current. This injection current is a triangular wave of nominal frequency $250 \mathrm{~Hz}$ having a $0.5-\mathrm{mA}$ peak-to-peak amplitude. The slope of laser frequency versus dc injection current is approximately $3 \mathrm{GHz} / \mathrm{mA}$. Since the Mach-Zehnder interferometer formed by beamsplitters 1 through 4 has arms of unequal path length $(\Delta L=0.38 \mathrm{~m})$, this frequency modulation induces a periodic time-varying phase difference between the two beams when they combine at the detector. The output of the detector thus consists of a dc photocurrent component for each beam and a single beat signal. By measuring the amplitude of both the beat signal and dc components, the MME may be calculated. Using (1), it is shown in Appendix B that

$$
\mathrm{MME}=\frac{I_{\mathrm{pp}}^{2}}{16\left[I_{1} \cdot I_{2}\right]}
$$

where

$I_{\mathrm{pp}}$ peak-to-peak beat signal photocurrent,

$I_{1}$ dc photocurrent produced by beam 1 ,

$I_{2}$ dc photocurrent produced by beam 2 .

Using the above technique, mode matching efficiencies of 90 percent (i.e., $0.46-\mathrm{dB}$ loss ) were obtained repeatably, and values as high as 92 percent were observed.

Figs. 5(a) and 5(b) show an intensity scan through beam 1 in the horizontal and vertical directions, respectively. Virtually identical results were obtained for beam 2. Each horizontal division in the figure represents a distance of $1.36 \mathrm{~mm}$, and thus the beam's $1 / e$ intensity point lies approximately $\sigma_{S}=1.84 \mathrm{~mm}$ from beam center. Also note that the intensity scan in the vertical direction exhibits local peak and troughs. These, we believe, are interference fringes generated by multiple reflections off of the laser collimating lens. The fringes do not occur in both the vertical and horizontal directions, because the beam leaving the semiconductor laser has different divergences in the two directions.

Fig. 6 shows an oscilloscope trace of the photocurrent beat signal generated at the output of the RCA p-i-n detector. The beam intensity striking the detector was controlled using neutral density filters so as not to saturate the photocurrent. Detector responsivity was measured as function of incident power, and the results are plotted in Fig. 7.

By using the LADITE to measure angular tilt $(\Delta \theta)$ between beams 1 and 2, we were also able to plot measured mode matching efficiencies versus $\Delta \theta$. Fig. 8 shows this plot along with the theoretically predicted curve derived

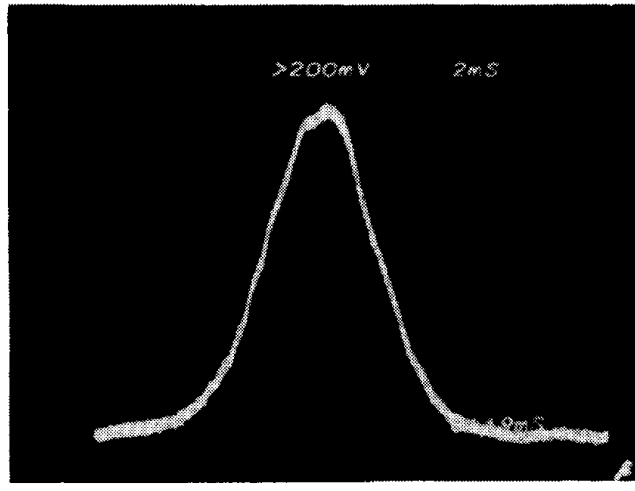

$1.36 \mathrm{~mm} /$ HORIZONTAL DIVISION

(a)

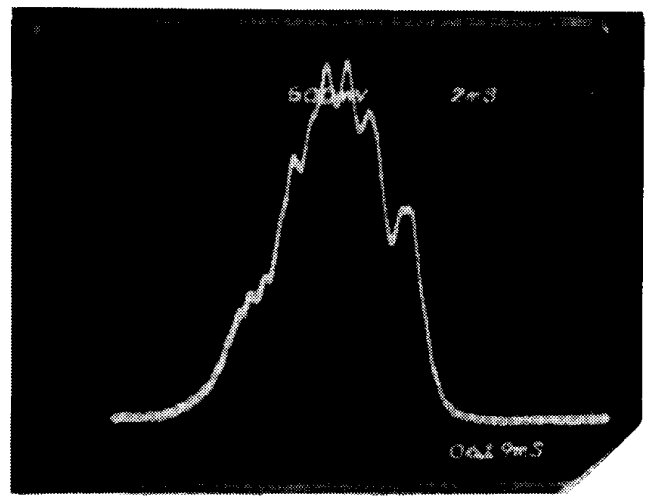

(b)

Fig. 5. (a) Horizontal intensity scan beam 1. (b) Vertical intensity scan beam 1 .

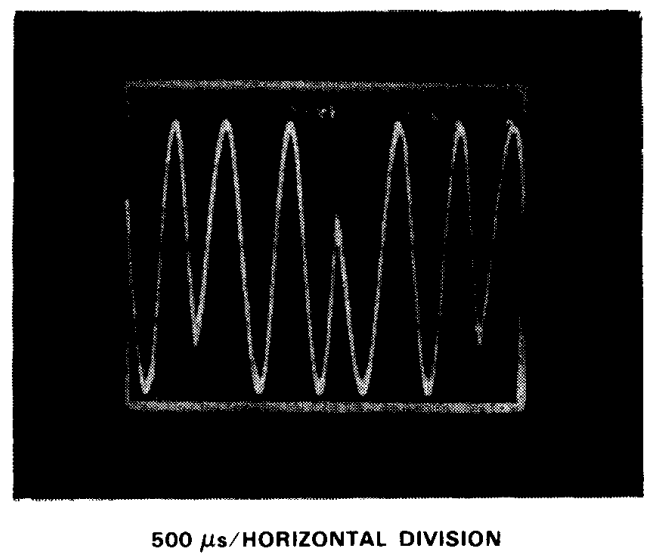

Fig. 6. Photocurrent beat signal (single laser case).

from (3). We note the excellent agreement between the theory and the measurements.

Under the best alignment conditions we were able to achieve, Fig. 8 indicates that approximately $-0.12 \mathrm{~dB}$ of mode matching loss can be attributed to a residual angular error of $17 \mu \mathrm{rad}$ between beams. We believe that most of the remaining $-0.4-\mathrm{dB}$ loss is due to the wavefront $\mathrm{ab}$ - 


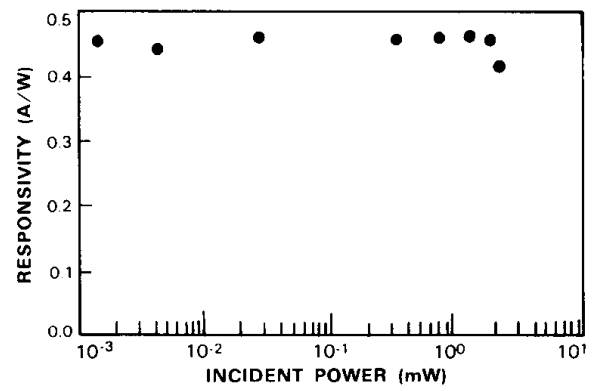

Fig. 7. Responsivity for RCA C30971E photodetector.

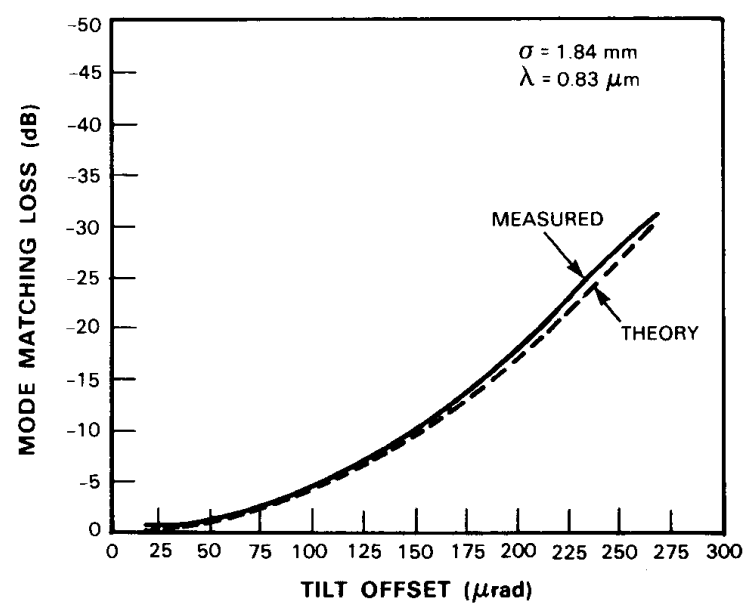

Fig. 8. Mode matching efficiency versus tilt offset.

errations introduced in the two different arms of the MachZehnder interferometer. It is also speculated that some of the loss is produced by laser frequency noise. Beams 1 and 2 follow paths which differ in length by approximately $0.38 \mathrm{~m}$ or $1.3 \mathrm{~ns}$ of propagation delay time. Consequently, frequency fluctuations which occur on this time scale or faster will reduce the amplitude of the beat signal photocurrent. For a Lorentzian laser linewidth of $\Delta \nu-\mathrm{Hz}$ full-width-half-maximum, it can be shown that the mean loss is given by the following multiplicative factor:

$$
\exp \left[-2 \pi \Delta \nu T_{D}\right]
$$

where $T_{D}$ is the propagation delay time between paths in seconds.

With $\Delta \nu=7 \mathrm{MHz}$ and $T_{D}=1.3 \times 10^{-9}$, (12) predicts a mean loss of $-0.24 \mathrm{~dB}$. Finally note by (4) that a 10 percent lateral offset (i.e., $\epsilon / \sigma_{S}=0.1$ ) between the centers of beams 1 and 2 would produce only a $-0.02-\mathrm{dB}$ loss. Thus, lateral offsets should not contribute significantly to measured losses.

Fig. 9 shows the experimental setup used to measure the mode matching efficiency when two separate lasers are heterodyned. Note that this setup is virtually identical to the one used for the self-heterodyned case. The two lasers were open-loop frequency stabilized as previously discussed and neither one was modulated. The two lasers

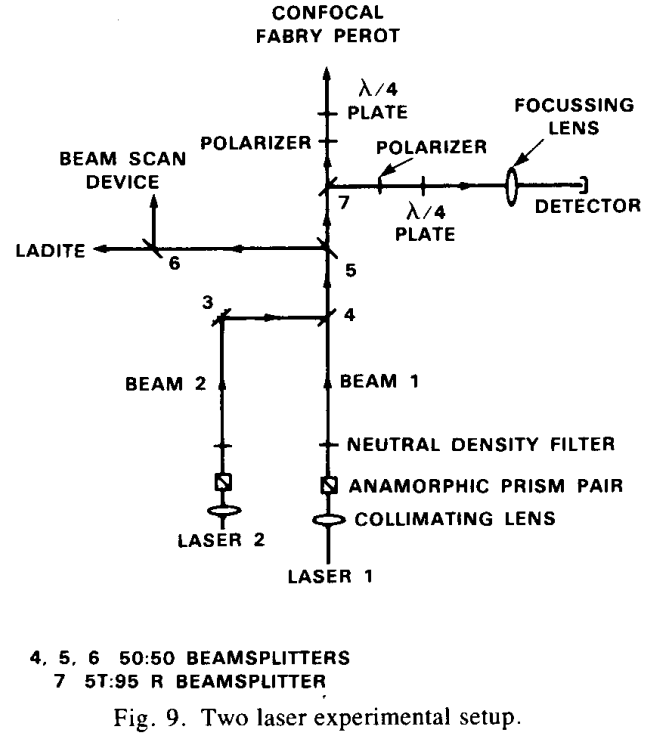

were frequency tuned by control of their temperatures and bias currents so that they would produce a heterodyned beat signal at an IF frequency of approximately $50 \mathrm{MHz}$. The frequency response of the detector and its associated packaging (including cable) was not measured in detail. A linear least squares fit, however, to ten data points collected between 25 and $400 \mathrm{MHz}$ indicates less than an $0.1-\mathrm{dB}$ roll-off at $50 \mathrm{MHz}$ with a $3-\mathrm{dB}$ point at $1.3 \mathrm{GHz}$.

The beat signal spectrum has a Lorentzian shape due to laser frequency noise, and its effect is quite pronounced. When the oscilloscope trace was triggered on a zero crossing of the IF beat signal, the resulting display was a fuzzy sinusoid as illustrated in Fig. 10. As expected the fuzziness increased from left to right across the scope screen. We chose to define the peak-to-peak IF photocurrent as the difference between the first minimum and maximum of the displayed signal. Using this peak-to-peak value along with (11), repeatable mode matching efficiencies of 75 percent (i.e., 1.25 -dB loss) were measured.

The accuracy of the above measurement was less than that for the self-heterodyned case for two main reasons. First, the high frequency oscilloscope plug-in used to observe the $50-\mathrm{MHz}$ beat signal had low sensitivity, thus limiting the accuracy with which the peak-to-peak signal values could be read. Second, as already discussed, frequency noise degraded the signal.

It is noted that the mode matching efficiency for the two laser case is approximately $0.8 \mathrm{~dB}$ worse than that recorded for self-heterodyning. At least some of this difference is due to the fact that the phase wavefronts of the two lasers (with tilt removed) are not identical, resulting in a loss as indicated by (10). In order to minimize this loss, the laser collimating lenses were adjusted with the help of the LADITE to produce wavefronts having low rms phase variations. Values of the order of $\lambda / 20$ were routinely achieved for each laser. Note that if the phase perturbations across each beam were random and inde- 


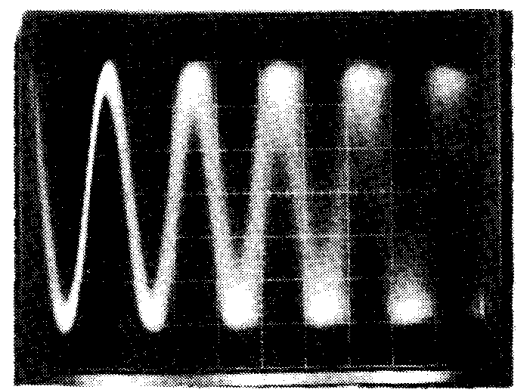

$2.5 \mathrm{~ns} /$ HORIZONTAL DIVISION

Fig. 10. Photocurrent beat signal (two laser case).

pendent, then the mean-squared phase difference between the two beams would be $2(2 \pi / 20)^{2} \mathrm{rad}^{2}$. According to (10), this value could result in a loss of as much as 0.95 $\mathrm{dB}$.

Spatial mode matching of optical beams is also of importance in applications other than free-space optical communications. These related applications include optical fiber heterodyne communications [12]-[14] and the coupling of beams into and out of resonators [15] and waveguides. As opposed to the configuration shown in Fig. 9, beam combining in fiber systems is usually accomplishd by launching both the LO and signal beams into a single-mode fiber or directional coupler [12]-[14]. The mode matching loss under these conditions becomes the coupler insertion loss, as seen by the signal beam. The beam combining techniques used in fiber systems are not practical for free space optical communications, because they do not permit heterodyne spatial tracking, and the insertion losses are likely to be high.

\section{Summary}

We have experimentally measured spatial mode matching efficiencies when heterodyning GaAlAs semiconductor lasers on high speed silicon $\mathrm{p}-\mathrm{i}-\mathrm{n}$ photodetectors. MME's were computed by comparing the peak-to-peak magnitude of the resulting beat signal with the dc photocurrents generated by each of the two beams. Two sets of measurements were taken. In the first, a frequency modulated GaAlAs laser was self-heterodyned using an unequal path length Mach-Zehnder interferometer, and MME values on the order of 90 percent were obtained. Approximately $0.1 \mathrm{~dB}$ of this loss was attributed to beam angular alignment errors of between 10 and $20 \mu \mathrm{rad}$. Alignment accuracies of this order were routinely achieved using a 300-MHz free-spectral-range confocal Fabry-Perot spectrum analyzer. The remaining $0.4 \mathrm{~dB}$ of loss is believed to result from wavefront aberrations produced by the Mach-Zehnder interferometer optics and measurement errors (including frequency noise effects).

In the second set of measurements, two separate GaAlAs lasers were heterodyned, and a $50-\mathrm{MHz}$ IF beat signal was produced. A constant $50-\mathrm{MHz}$ IF frequency was maintained by open-loop frequency stabilizing each laser through control of its temperature and bias current. The output of each laser was collimated to approximately $\lambda / 20$ rms, and spatial mode matching efficiencies of 75 percent were measured. As expected, the measured MME for the two laser case was less than that obtained during self-heterodyning. Some of this difference is due to wavefront mismatch, and the remainder can be attributed to an increase in measurement error. The measurement error increase results from the pronounced effects of frequency noise, and the reduced sensitivity of the oscilloscope's plug-in amplifier at $50 \mathrm{MHz}$.

\section{APPENDIX A \\ Derivation of Mode Matching Efficiency Lower BOUND}

In this appendix a lower bound on the mode matching efficiency will be derived, assuming a small phase mismatch exists between the $\mathrm{LO}$ and signal beams.

Let $\Delta \phi(x)$ denote the phase difference between the beams. Then

$$
\Delta \phi(x) \triangleq \phi_{s}(x)-\phi_{\mathrm{LO}}(x)
$$

Also, without loss of generality, let $\Delta \phi(x)$ have an average value of zero as indicated below:

$$
\iint \Delta \phi(x) d x \equiv 0
$$

Next, assume that the phase difference $\Delta \phi(x)$ is small or equivalently

$$
|\Delta \phi(\boldsymbol{x})| \ll 1 .
$$

The mean-squared phase difference (MSE) between the LO and signal beams is defined by

$$
\mathrm{MSE} \triangleq \frac{\iint \Delta \phi^{2}(x) d x}{\iint d x}
$$

In addition, let $B$ be defined by (A5) below:

$$
B \triangleq \iint A_{s}^{2}(x) d x .
$$

Then, from (1) in the text it follows that

$$
\mathrm{MME}_{\text {phase }}=B^{-2}\left|\iint A_{s}^{2}(\boldsymbol{x}) \exp [i \Delta \phi(x)] d x\right|^{2} \text {. }
$$

Expressing the exponential in (A6) by the first three terms in its series expansion yields

$\mathrm{MME}_{\text {phase }}=B^{-2}\left|\iint A_{s}^{2}(x)\left[1+i \Delta \phi(x)-\Delta \phi^{2}(x) / 2\right]\right|^{2}$

$$
\geq B^{-2}\left|\iint A_{s}^{2}(\boldsymbol{x})\left[1-\Delta \phi^{2}(\boldsymbol{x}) / 2\right] d x\right|^{2}
$$




$$
\begin{aligned}
& =\left|1-B^{-1} \iint A_{s}^{2}(x)\left(\Delta \phi^{2}(x) / 2\right) d x\right|^{2} \\
& \geq 1-B^{-1} \iint A_{s}^{2}(x) \Delta \phi^{2}(x) d x
\end{aligned}
$$

By the Schwarz Inequality the integral in (A10) is maximum when

$$
\Delta \phi^{2}(x)=c A_{s}^{2}(x), \quad \text { for } c \text { a constant. }
$$

Therefore

$$
\mathrm{MME}_{\text {phase }} \geq 1-c B^{-1} \iint A_{s}^{4}(x) d x
$$

where by (A11)

$$
\iint \Delta \phi^{2}(x) d x=c \iint A_{s}^{2}(x) d x .
$$

Combining (A12) and (A13) yields

$$
\begin{aligned}
\mathrm{MME}_{\text {phase }} \geq & 1-B^{-2} \iint \Delta \phi^{2}(x) d x \iint A_{s}^{4}(x) d x \\
= & 1-\frac{\iint \Delta \phi^{2}(x) d x}{\iint d x} B^{-2} \\
& \cdot \iint A_{s}^{4}(x) d x \iint d x .
\end{aligned}
$$

By the Schwarz Inequality

$$
B^{2} \leq \iint A_{s}^{4}(x) d x \iint d x
$$

Combining (A4), (A15), and (A16) produces the final desired result

$$
\mathrm{MME}_{\text {phase }} \geq 1-\mathrm{MSE} \text {. }
$$

APPENDIX B

Derivation of a Mode Matching Efficiency Expression in Terms of Observed Photocurrents

In this appendix, an expression for mode matching efficiency is derived in terms of observed photocurrents. Let the analytic forms of the two beams (i.e., LO and signal) be given as:

Beam 1:

$$
A_{1}(x) \exp \left[i \omega_{1} t+i \phi_{1}(x)\right]
$$

Beam 2:

$$
A_{2}(\boldsymbol{x}) \exp \left[i \omega_{2} t+i \phi_{2}(\boldsymbol{x})\right] \text {. }
$$

Define $\Delta \omega, \Delta \phi(x), P, Q$, and $R$ by

$$
\begin{aligned}
\Delta \omega & \triangleq \omega_{1}-\omega_{2} \\
\Delta \phi(x) & =\phi_{1}(x)-\phi_{2}(x) \\
P & \triangleq \iint A_{1}(x) A_{2}(x) \cos [\Delta \phi(x)] d x \\
Q & \triangleq \iint A_{1}(x) A_{2}(x) \sin [\Delta \phi(x)] d x \\
R & =\text { detector responsivity in amperes per watt. }
\end{aligned}
$$

Thus, the dc photocurrents are

$$
\begin{aligned}
& I_{1}=\frac{1}{2} R \iint A_{1}^{2}(x) d x \\
& I_{2}=\frac{1}{2} R \iint A_{2}^{2}(x) d x
\end{aligned}
$$

and the beat signal current $i(t)$ is given by

$$
\begin{aligned}
i(t)= & R \iint A_{1}(x) A_{2}(x) \\
& \cdot \cos \left[\left(\omega_{1}-\omega_{2}\right) t+\phi_{1}(x)-\phi_{2}(x)\right] d x \\
= & \cos (\Delta \omega t) R \cdot P-\sin (\Delta \omega t) R \cdot Q
\end{aligned}
$$

$i(t)$ will achieve a maximum or minimum when $d i(t) / d t$ $=0 . d i(t) / d t=0$ implies

$$
-\sin (\Delta \omega t) P-\cos (\Delta \omega t) Q=0
$$

or equivalently

$$
\sin (\Delta \omega t)= \pm \frac{Q}{\left(P^{2}+Q^{2}\right)^{1 / 2}}
$$

and

$$
\cos (\Delta \omega t)=\mp \frac{P}{\left(P^{2}+Q^{2}\right)^{1 / 2}} .
$$

Therefore by (B11) and (B13)

$$
\begin{aligned}
\max i(t) & =R\left(P^{2}+Q^{2}\right)^{1 / 2} \\
\min i(t) & =-R\left(P^{2}+Q^{2}\right)^{1 / 2} \\
I_{p-p} & =2 R\left(P^{2}+Q^{2}\right)^{1 / 2} .
\end{aligned}
$$

Combining (B1)-(B6), (B8), (B9), and (B16) yields the final desired result

$$
\begin{aligned}
\frac{I_{p-p}^{2}}{16 I_{1} I_{2}} & =\frac{\left|\iint A_{1}(x) \exp \left[i \phi_{1}(x)\right] A_{2}(x) \exp \left[-i \phi_{2}(x)\right] d x\right|^{2}}{\iint A_{1}^{2}(x) d x \iint A_{2}^{2}(x) d x} \\
& =\text { MME. }
\end{aligned}
$$




\section{REFERENCES}

[1] G. L. Abbas, S. B. Alexander, R. S. Bondurant, V. W. S. Chan, J. M. Elder, L. L. Jeromin, J. E. Kaufmann, S. D. Lowney, D. v. L. Marquis, F. G. Walther, D. Welford, and S. T. K. Yee, "Near-quantum-limited operation of a GaAlAs laser heterodyne communications system," presented at Conf. Lasers and Electro-Optics, Baltimore, MD, May 1985.

[2] J. J. Degnan and B. J. Klein, "Optical antenna gain 2: receiving antennas," Appl. Opt., vol. 13, pp. 2397-2401, Oct. 1974

[3] D. Fink, "Coherent detection signal-to-noise," Appl. Opt., vol. 14, pp. 689-690, Mar. 1975

[4] S. C. Cohen, "Heterodyne detection: Phase front alignment, beam spot size, and detector uniformity," Appl. Opt., vol. 14, pp. $1953-$ 1959, Aug. 1975.

[5] L. Mandel and E. Wolf, "Optimum conditions for heterodyne detection of light," J. Opt. Soc. Amer., vol. 65, pp. 413-420, Apr. 1975.

[6] N. Saga, K. Tanaka, and O. Fukumitsu, "Diffraction of a Gaussian beam through a finite aperture lens and the resulting heterodyne efficiency," Appl. Opt., vol. 20, pp. 2827-2831, Aug. 1981

[7] K. Tanaka and N. Ohta, "Effects of tilt and offset of signal field on heterodyne efficiency,' Appl. Opt., vol. 26, pp. 627-632, Feb. 1987.

[8] H. E. Sonntag, "Experimental verification of the optimum conditions for heterodyne detection of optical fields," Opt. Commun., vol. 18, pp. 546-550, Sept. 1976.

[9] D. L. Fried, "Optical heterodyne detection of an atmospherically distorted signal wave front." Proc. IEEE, vol. 55, pp. 57-66, Jan. 1967.

[10] J. Hayes and S. Lange, "A heterodyne interferometer for testing laser diodes," Proc. Soc. Photo. Opt. Instrum. Eng., vol. 429, pp. 22-26, Aug. 1983.

[11] C. R. Munnerlyn and J. W. Balliett, "Alignment requirements for mode matching in a confocal Fabry-Perot inte-ferometer," Appl. Opt., vol. 9, pp. 2535-2538, Nov. 1970.

[12] Y. Yamamoto and T. Kimura, "Coherent optical fiber transmission systems," IEEE J. Quantum Electron., vol. QE-17, pp. 919-935, June 1981 .

[13] F. Favre, L. Jeunhomme, I. Joindot, M. Monerie, and J. C. Simon, "Progress towards heterodyne-type single-mode fiber communications systems, " IEEE J. Quantum Electron., vol. QE-17, pp. $897-$ 906 , June 1981

[14] S. Saito, Y. Yamamoto, and T. Kimura, "Optical FSK heterodyne detection experiments using semiconductor laser transmitter and local oscillator," IEEE J. Quantum Electron., vol. QE-17, pp. 935-941, June 1981.

[15] H. Kogelnik and T. Li, "Laser beams and resonators," Proc. IEEE, vol. 54, pp. 1312-1329, Oct. 1966.

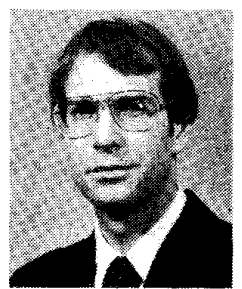

Kim A. Winick was born in New York City on July 27,1954 . He received the B.S. degree in electrical engineering from The Pennsylvania State University in 1976 , and the M.S. and Ph.D. degrees in electrical engineering from the University of Michigan in 1977 and 1981 , respectively. His Ph.D. dissertation topic, supervised by Prof. E. Leith, was a study of optical propagation through structures having quasisinusoidal refractive index profiles. While at the University of Michigan, he was a National Science Foundation Graduate Fellow.

Since 1981, he has worked in the Satellite Communications Division at the Massachusetts Institute of Technology Lincoln Laboratory, currently as a member of the Optical Communications Technology Group. He has conducted research in the areas of spatial mode matching measurements with semiconductor lasers, atmospheric propagation studies and experiments, statistical estimation theory, holography, and corrugated waveguide filter design.

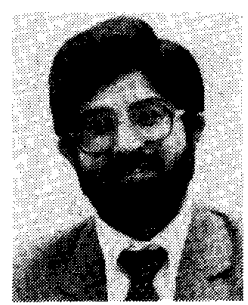

Prem Kumar was born in New Delhi, India, on September 16, 1955. He received the B.Sc. in physics (honors) from the University of Delhi in 1974 , the M.Sc. in physics from the Indian Institute of Technology at Kanpur in 1976, and the Ph.D. in physics from the State University of New York at Buffalo in 1980. His Ph.D. dissertation topic was an experimental study of the process of intracavity absorption in a dye laser using an atomic beam of barium as the probe absorber.

After spending a year at the Center for Laser Studies at the University of Southern California, he joined the Research Laboratory of Electronics at the Massachusetts Institute of Technology in 1981. He was also a part-time Lecturer in the Department of Electrical Engineering and Computer Science during 1984-1985 and a Staff Scientist at the M.I.T. Lincoln Laboratory during 1986. Since the Fall of 1986, he has been an Associate Professor in the Department of Electrical Engineering and Computer Science at Northwestern University.

Dr. Kumar's major research interest has been in the generation and application of squeezed-light and he has published extensively in this area. His other research interests include nonlinear optics, lasers, atomic physics, time/frequency standards, and coherent optical communications. He is a member of the American Physical Society and the Optical Society of America. 